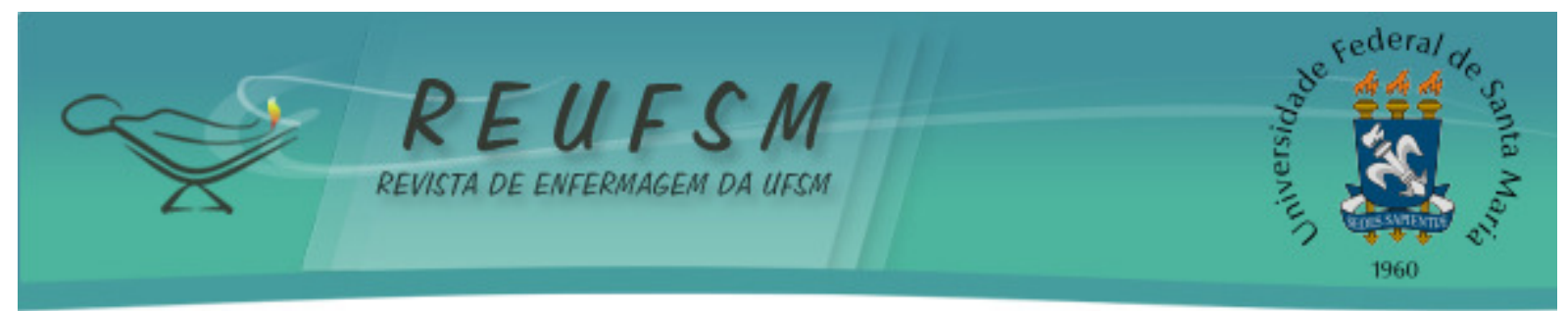

ARTIGO ORIGINAL

\title{
USO DE SOFTWARE GRATUITO COMO RECURSO PARA MENSURAÇÃO DE ÁREA DE ÚLCERAS VENOSAS
}

\author{
THE USE OF FREE SOFTWARE AS A RESOURCE FOR VENOUS ULCERS \\ AREA MEASUREMENT
}

USO DE SOFTWAREGRATUITO COMO RECURSO PARA MENSURACIÓN DE ÁREA DE ÚLCERAS VENOSAS

Doi: $10.5902 / 2179769227611$

\author{
Thaís Dresch Eberhardt ${ }^{1}$ \\ Suzinara Beatriz Soares de Lima ${ }^{2}$ \\ Luis Felipe Dias Lopes ${ }^{3}$ \\ Dienifer Fortes da Fonseca ${ }^{4}$ \\ Jordana Lopes Carvalho 5 \\ Graziele Gorete Portella da Fonseca ${ }^{6}$
}

RESUMO: Objetivo: verificar a reprodutibilidade do uso do software Image tool para mensuração em área de úlceras venosas. Método: estudo de avaliação de reprodutibilidade de testes realizado em ambulatório de angiologia de hospital universitário do sul do Brasil. Foram selecionados pacientes com úlceras venosas, os quais tiveram suas úlceras fotografadas e mensuradas por meio do software supracitado. Os dados foram analisados estatisticamente. Resultados: não se observou diferença $(\mathrm{p}=0,51)$ entre as mensurações. Observaram-se valores excelentes de Coeficiente de Correlação Intraclasse e Coeficiente de Correlação de Concordância. O software parece ser mais preciso quando utilizado para mensurar feridas grandes (com área $>10 \mathrm{~cm}$ ). Conclusão: o software é reprodutível para mensuração de área de úlceras venosas, sendo mais preciso para mensurar úlceras venosas com área $>10 \mathrm{~cm}^{2}$.

Descritores: Enfermagem; Úlcera varicosa; Pesos e medidas; Validação de programas de computador; Informática em enfermagem.

ABSTRACT: Aim: to verify the reproducibility of the Image tool software use for measuring venous ulcers area. Method: reproducibility assessment study of tests conducted in an angiology outpatient clinic of a university hospital in southern Brazil. Patients with venous ulcers were selected, having their ulcers photographed and measured using the above mentioned software. Data were statistically analysed. Results: no difference $(p=0.51)$ was observed between measurements. Excellent values of Intraclass Correlation Coefficient and

\footnotetext{
${ }^{1}$ Enfermeira, Mestre em Enfermagem, Doutoranda do Programa de Pós-Graduação em Enfermagem, Universidade Federal de Santa Maria, Santa Maria, RS, Brasil. E-mail: thaiseberhardt@gmail.com.

${ }^{2}$ Enfermeira, Doutora em Enfermagem, Professora Associada, Universidade Federal de Santa Maria, Santa Maria, RS, Brasil. E-mail: suzibslima.com.br.

${ }^{3}$ Licenciado em Matemática, Doutor em Engenharia da Produção, Professor Titular, Universidade Federal de Santa Maria, Santa Maria, RS, Brasil. E-mail: lflopes67@yahoo.com.br.

${ }^{4}$ Enfermeira, Mestranda do Programa de Pós-Graduação em Enfermagem, Universidade Federal de Santa Maria, Santa Maria, RS, Brasil. E-mail: dieni_ff@yahoo.com.br.

${ }^{5}$ Enfermeira, Mestranda do Programa de Pós-Graduação em Enfermagem, Universidade Federal de Santa Maria, Santa Maria, RS, Brasil. E-mail: dana.lopes@ hotmail.com.

${ }^{6}$ Enfermeira, Mestranda do Programa de Pós-Graduação em Enfermagem, Universidade Federal de Santa Maria, Santa Maria, RS, Brasil. E-mail: grazieleportelladafonseca@gmail.com.
} 


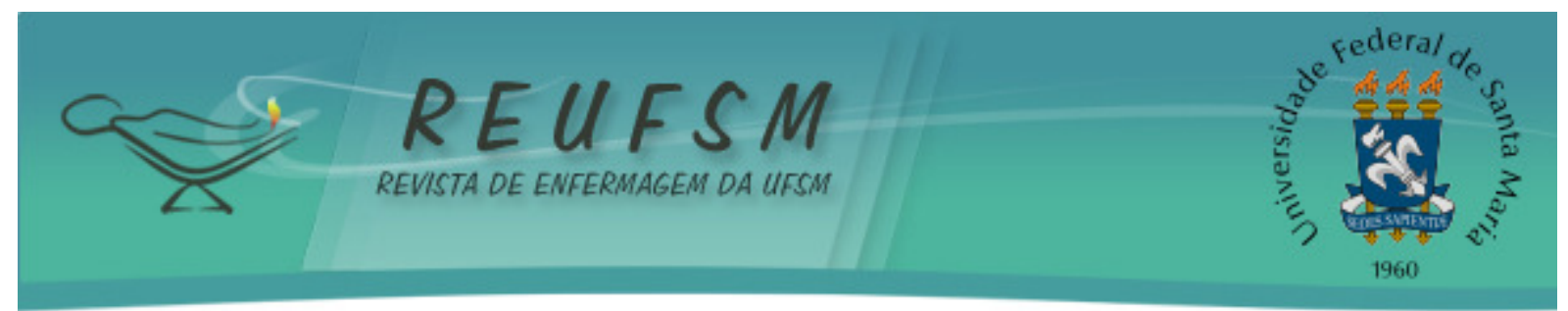

Concordance Correlation Coefficient were observed. The software seems to be more accurate when used to measure large wounds (area $>10 \mathrm{~cm}$ ). Conclusion: Image tool software is reproducible for measuring venous ulcers area, being more precise to measure venous ulcers with area $>10 \mathrm{~cm}^{2}$.

Descriptors: Nursing; Varicose ulcer; Weights and measures; Software validation; Nursing informatics.

RESUMEN: Objetivo: verificar la reproducibilidad del uso del software Image tool para medir el área de úlceras venosas. Método: estudio de evaluación de reproducibilidad de pruebas realizado en ambulatorio de angiología de un hospital universitario del sur de Brasil. Fueron seleccionados pacientes con úlceras venosas, que tuvieron sus úlceras fotografiadas y mensuradas por medio del software anteriormente citado. Los datos fueron analizados estadísticamente. Resultados: no se observó diferencia $(p=0,51)$ entre las mediciones. Se observó valores excelentes de Coeficiente de Correlación Intraclase y Coeficiente de Correlación de Concordancia. El software parece ser más preciso cuando utilizado para medir heridas más grandes (con área>10 cm). Conclusión: el software es reproducible para la medición de área de úlceras venosas, pero es más preciso para medir úlceras venosas con área $>10 \mathrm{~cm}^{2}$.

Descriptores: Enfermería, Úlcera varicosa, Pesos y medidas; Validación de programas de computación; Informática aplicada a la enfermería.

\section{INTRODUÇÃO}

Úlcera venosa pode ser definida como uma lesão aberta da pele na perna ou pé, que ocorre em área afetada pela hipertensão venosa. ${ }^{1}$ Essas lesões podem afetar a condição de vida dos pacientes, pois são um agravo crônico, social e econômico, além de representar um desafio para os profissionais de saúde. Estes pacientes podem apresentar dor, dificuldade de mobilidade, redução da autoestima, necessitando de cuidado integral e multiprofissional, visando à melhoria da qualidade de vida e o acesso facilitado nos serviços de saúde. ${ }^{2}$

Dessa maneira, conhecer os pacientes com úlcera venosa em seus aspectos clínicos e biopsicossociais, possibilita contribuir para o planejamento dos cuidados de enfermagem, direcionando para as necessidades reais do cuidado. ${ }^{3}$ Nesse sentido, a avaliação das lesões faz parte do processo de cuidado e tratamento das mesmas. A mensuração de feridas é um procedimento fundamental na avaliação das úlceras venosas, $^{1,4-5}$ pois permite acompanhar a evolução do tamanho da ferida e avaliar se a intervenção utilizada está sendo eficaz.

Em estudo realizado com o objetivo de caracterizar os protocolos de assistência aos portadores de feridas existentes no Brasil, foram analisados oito trabalhos, sendo que três indicaram a mensuração de feridas como parte integrante da avaliação. ${ }^{6} \mathrm{O}$ processo de 


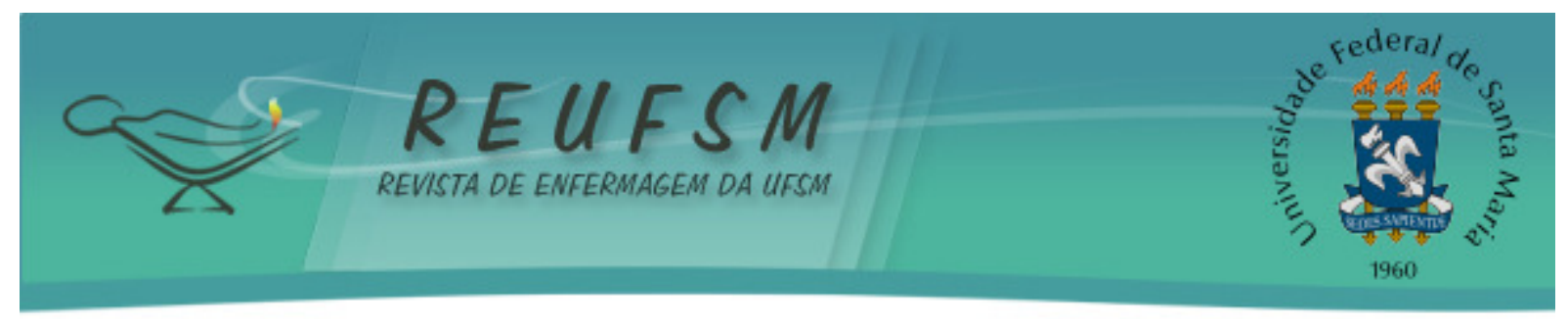

mensuração é considerado etapa importante na tomada de decisão do plano terapêutico, permitindo o monitoramento e intervenção no processo de cicatrização. ${ }^{7}$

Para realizar realizá-la, podem ser utilizadas fotografias digitais e softwares específicos, como o Image Tool. Este foi desenvolvido pela University of Texas Health Sciences Center at San Antonio, é um software gratuito, apresentado como um instrumento prático, capaz de obter medidas objetivas, fidedignas ao tamanho real da lesão, conferindo segurança, rapidez e confiabilidade ao processo de mensuração. ${ }^{8}$

Além das fotografias digitais e softwares, considerados métodos não invasivos de mensuração por evitarem o contato direto com as lesões, existem métodos considerados invasivos. Dentre esses, destacam-se o uso da régua e da folha de acetato. Os métodos invasivos possuem algumas limitações, como risco de lesão tecidual, contaminação local e de infecção. ${ }^{9}$

Em revisão da literatura, identificou-se a necessidade de pesquisas que utilizem métodos não invasivos de mensuração, como as fotografias digitais e os softwares, ${ }^{9}$ justificando a escolha deste, além de ser gratuito. Ainda, a pesquisa se justifica na medida em que o uso de régua para mensuração da área de superfícies é contraindicada, pois tende a superestimar o seu tamanho. ${ }^{10}$ Os métodos de mensuração de úlceras venosas nos estudos não são precisos, apresentando uma lacuna no conhecimento. ${ }^{11}$

Diante do exposto, elaborou-se a seguinte questão de pesquisa: o software Image tool permite a mensuração fidedigna de áreas de úlceras venosas?Tem-se como objetivo verificar a reprodutibilidade do uso do software Image tool para mensuração em área de úlceras venosas.

\section{MÉTODO}

Trata-se de um estudo de avaliação de reprodutibilidade de testes. Esta se refere a consistência ou concordância de resultados, quando a mensuração se repete em condições idênticas. ${ }^{12}$ Foi realizado em um ambulatório de angiologia de um hospital universitário do sul do Brasil, que atende pacientes encaminhados pela Coordenadoria Regional de Saúde ou pelo próprio hospital e por demanda espontânea.

Foram incluídos todos os pacientes portadores de úlceras venosas, atendidos no ambulatório no período de coleta de dados (março a julho de 2015), com idade superior a 18 


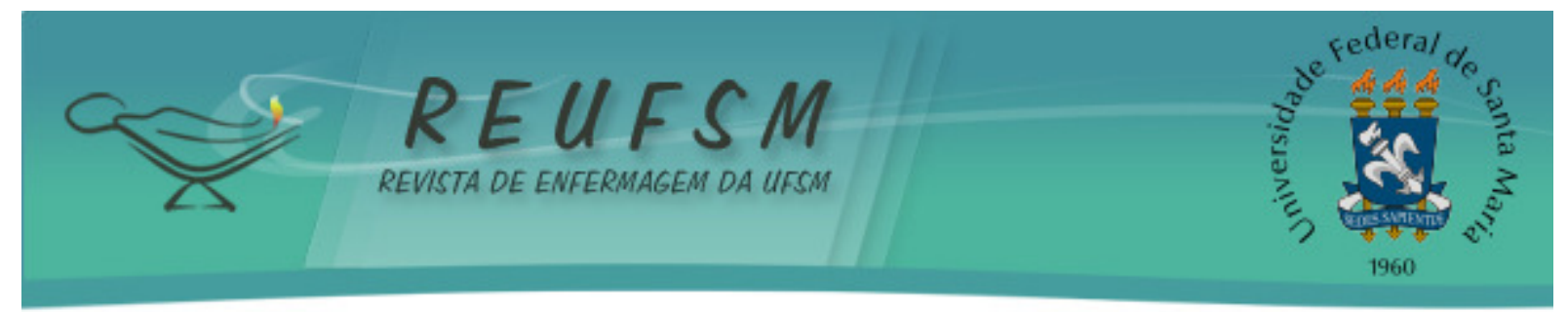

anos, com capacidade cognitiva e verbal preservadas, que possuíam úlceras cobrindo uma face do membro inferior (21 pacientes com 36 úlceras venosas).

Para o procedimento de coleta de dados, cinco enfermeiras participantes do Grupo de Estudos de Lesões de Pele do hospital foram colaboradoras da pesquisa, participando deste processo e sendo denominados de avaliadoras. Todas receberam treinamento sobre o processo de obtenção das fotografias e da mensuração das feridas.

As úlceras venosas foram fotografadas pela pesquisadora e por uma das enfermeiras (avaliadoras) capacitadas, que estavam presentes no momento da realização do curativo. Ambas as fotografias foram realizadas durante o mesmo atendimento.

Após, as fotografias foram transferidas para um notebook pessoal da pesquisadora. Tanto as avaliadoras quanto a pesquisadora realizaram as mensurações das duas fotografias. Este processo foi executado com todas as avaliadoras, sempre em pares (pesquisadora e uma avaliadora), até atingir o número máximo de indivíduos pertencentes à população em estudo (36 úlceras, 72 fotografias, 144 mensurações).

Para o procedimento de coleta de dados, foram seguidos os protocolos a seguir:

a) Protocolo para obtenção das fotografias (após a realização da limpeza da ferida com soro fisiológico $0,9 \%$ ): foi utilizada compressa embaixo do membro inferior, de modo que o fundo da fotografia ficasse da cor branca; objeto de referência (quadrado na cor preta impresso em folha A4, com três centímetros de lado) foi colocado no campo branco; a fotografia foi obtida com Câmera FujufilmFinepixS 14 Mega pixels (abertura f/6.4, ISO 400, função macro habilitada, white balance automático, nitidez alta, flash desligado), posicionando-se a câmera perpendicularmente à ferida (ângulo de $90^{\circ}$ ) e a $50 \mathrm{~cm}$ da ferida, aproximando-a ou afastando-a da ferida, conforme a necessidade (Figura 1a);

b) Protocolo para mensuração da área da ferida ${ }^{13}$ (Figura 1b). 


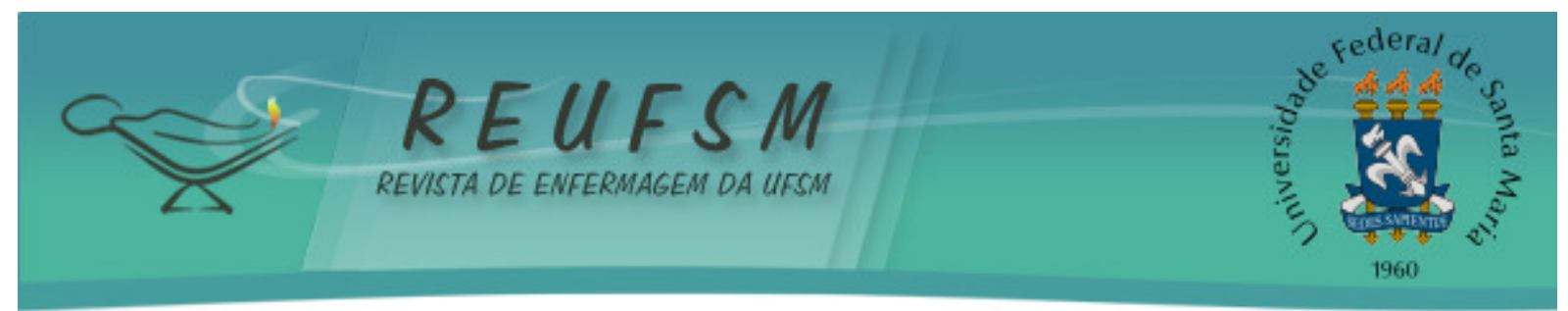

Figura 1 - a) Fotografia de úlcera venosa; b) Mensuração da área no software Image toolversão 3.0. Santa Maria, Rio Grande do Sul, Brasil, 2015.

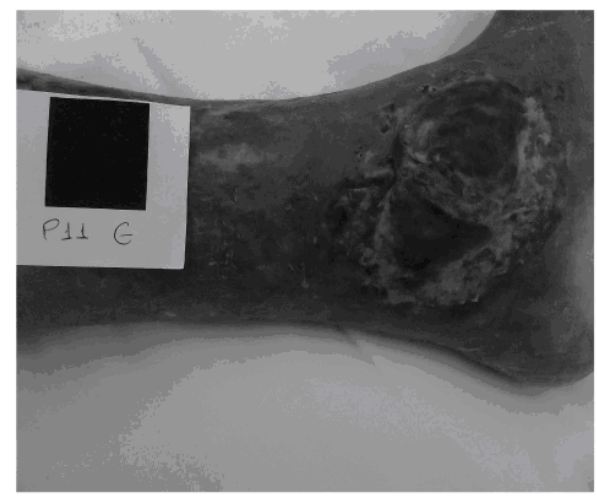

(a)

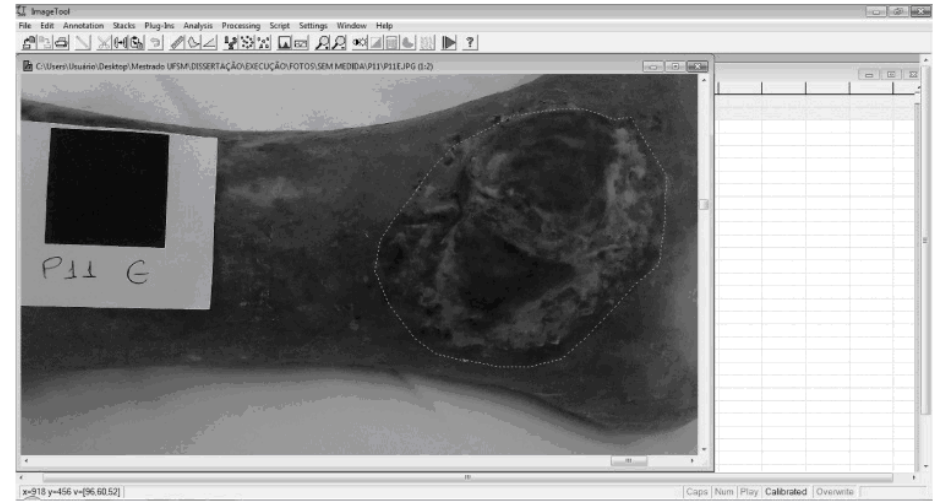

(b)

Os dados foram digitados de forma dupla e independente no Microsoft Office Excel®, a fim de corrigir possíveis falhas de digitação e analisados com auxílio do programa estatístico R.

Para o tratamento estatístico da variável área das úlceras venosas, foram utilizados média e erro padrão. A fim de identificar a distribuição de normalidade dos dados, aplicou-se o teste Shapiro Wilk e devido a não normalidade dos dados, utilizou-se teste de Wilcoxon, buscando verificar o grau de diferença sistemática entre as mensurações em pares (pesquisadora e avaliadora).

Ainda, foi analisada a distribuição das diferenças entre as medidas e a diferença média entre elas. ${ }^{14} \mathrm{~A}$ reprodutibilidade foi aferida por meio do Coeficiente de Correlação Intraclasse (CCI) e Coeficiente de Correlação de Concordância (CCC) de Lin. ${ }^{15-16}$ A correlação foi considerada baixa para valores $<0,40$, moderada entre 0,40 e 0,75 e excelente para valores $>0,75$.

O procedimento de Bland e Altman, ${ }^{17}$ foi realizado somente com os dados que apresentaram distribuição normal após transformações logarítmicas. Para efeito de análise, utilizou-se um nível de significância de 5\% e Intervalo de Confiança (IC) de 95\%.

Os preceitos éticos foram baseados na Resolução do Conselho Nacional de Saúde $\mathrm{n}^{\circ}$ 466 de 12 de dezembro de 2012. O projeto foi submetido à apreciação de Comitê de Ética de Pesquisa, sendo aprovado sob parecer $\mathrm{n}^{\circ} 932.838$ e Certificado de Apresentação para Apreciação Ética (CAAE) $n^{\circ}$ 40250814.6.0000.5346. A pesquisa procedeu mediante assinatura do Termo de Consentimento Livre e Esclarecido pelos participantes. 


\section{RESULTADOS}

As áreas mensuradas pela pesquisadora variaram de 0,3 a $89,1 \mathrm{~cm}^{2}$, tendo média de 13,6 $\pm 2,0 \mathrm{~cm}^{2}$. Já as áreas mensuradas pelas avaliadoras variaram de 0,4 a 68,2 $\mathrm{cm}^{2}$, tendo média de $16,1 \pm 2,3 \mathrm{~cm}^{2}$. A diferença média entre as mensurações da pesquisadora e das avaliadoras foi de $3,0 \pm 0,6 \mathrm{~cm}^{2}$.

Analisando os resultados da mensuração, não se observou diferença $(\mathrm{p}=0,51)$ entre as mensurações realizadas pela pesquisadora e pelas avaliadoras, sendo que a dispersão dos dados pode ser visualizada na Figura 2.

Figura 2 - a) Gráfico de dispersão das áreas mensuradas pela pesquisadora(area1) e avaliadoras (area9); b) Gráfico de dispersão das áreas $\leq 10 \mathrm{~cm}^{2}$ mensuradas pela pesquisadora (area1) e pelas avaliadoras (area9); c) Gráfico de dispersão das áreas $>10 \mathrm{~cm}^{2}$ mensuradas pela pesquisadora (area1) e pelas avaliadoras (area9), Santa Maria, Rio Grande do Sul, Brasil, 2015.

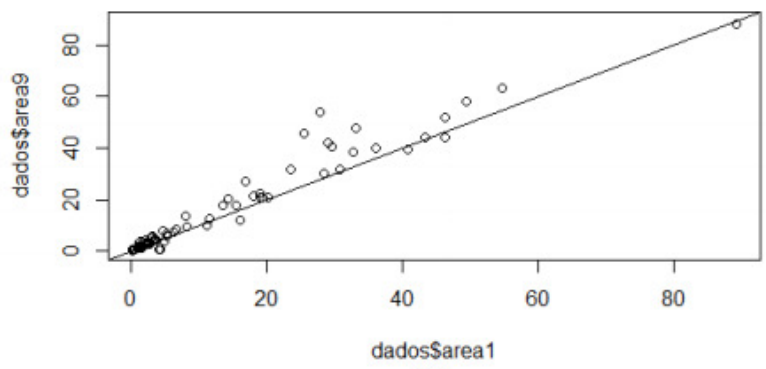

(a)

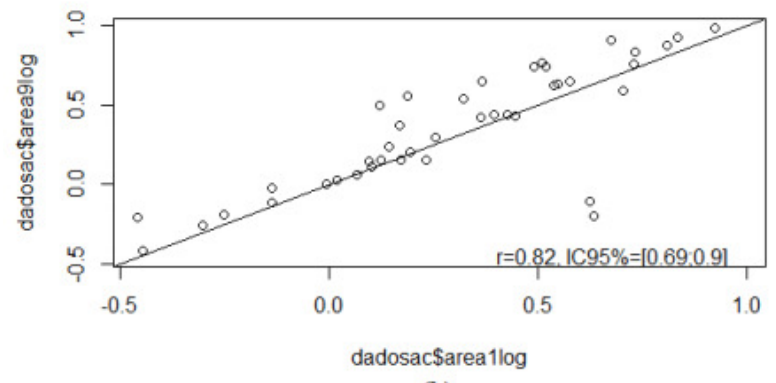

(b)

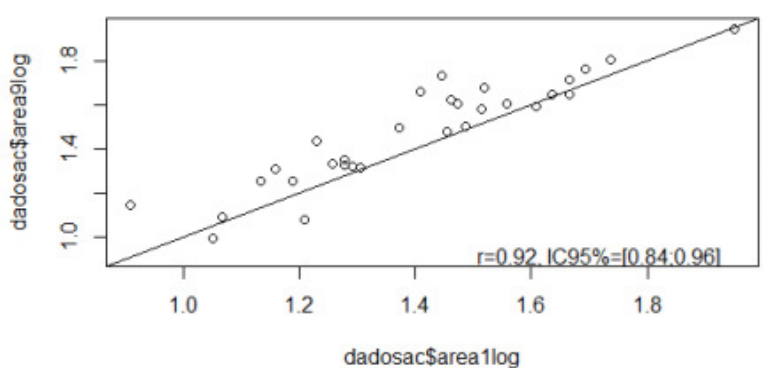

(c)

Em relação ao CCI referente às duas medidas, observou-se valor excelente $[\rho=0,97$; IC 95\% $(0,95-0,98) ; \mathrm{p}<0,05]$. Ao calcular o CCC, também obteve-se correlação excelente $[\mathrm{CCC}=0,95 ;$ IC $95 \%(0,93-0,97)$. 


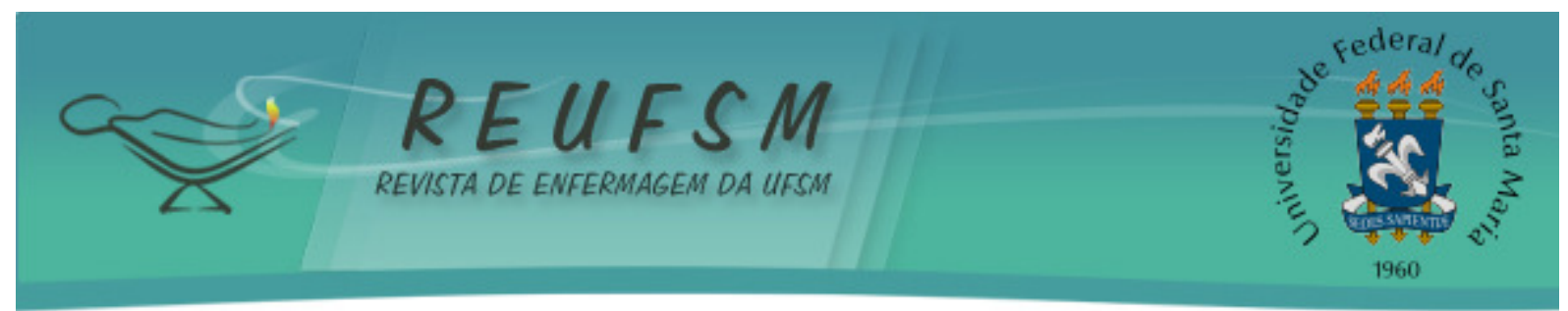

Não se observou diferença entre as medidas das úlceras venosas tanto com área $\leq 10$ $\mathrm{cm}^{2}(\mathrm{p}=0,51)$, quanto com área $>10 \mathrm{~cm}^{2}(\mathrm{p}=0,19)$. Os valores de CCI e CCC foram excelentes para todas as comparações, conforme apresentado na Tabela 1.

Tabela 1 - Coeficiente de correlação intraclasse e Coeficiente de correlação de concordância das úlceras venosas mensuradas no softwareImage Tool. Santa Maria, RS, Brasil, 2015.

\begin{tabular}{llll}
\hline \multicolumn{1}{c}{ Mensurações } & \multicolumn{1}{c}{$\mathbf{C C I}^{(\mathbf{1})}(\mathbf{I C ~ 9 5 \%})^{(2)}$} & p-valor $^{(3)}$ & $\mathbf{C C C}^{(4)}(\mathbf{I C ~ 9 5 \%})^{(2)}$ \\
\hline Área $\leq 10 \mathrm{~cm}^{2}$ & $0,82(0,69-0,90)$ & $<0,05$ & $0,80(0,68-0,88)$ \\
Área $>10 \mathrm{~cm}^{2}$ & $0,92(0,84-0,96)$ & $<0,05$ & $0,89(0,78-0,94)$ \\
\hline
\end{tabular}

1: Coeficiente de correlação intraclasse.

2: Intervalo de confiança.

3: p-valor da correlação intraclasse.

4: Coeficiente de correlação de concordância.

O gráfico de Bland e Altman (Figura 3) apresenta a concordância entre as mensurações. Este foi realizado para as duas categorias (úlceras $\leq 10 \mathrm{~cm}^{2}$ e $>10 \mathrm{~cm}^{2}$ ), pois foram os dados que apresentaram distribuição normal após transformação logarítmica.

Os limites apresentados nos gráficos estão transformados logaritmicamente, portanto o limite inferior de concordância (LIC) das mensurações das úlceras venosas com até $10 \mathrm{~cm}^{2} \mathrm{de}$ área é de $0,32 \mathrm{~cm}^{2}$, e limite superior de concordância (LSC) de $2,45 \mathrm{~cm}^{2}$. Quanto às úlceras com área maior que $10 \mathrm{~cm}^{2}$, obteve-se LIC de $0,55 \mathrm{~cm}^{2}$ e LSC de $1,29 \mathrm{~cm}^{2}$.

Figura 3 - a) Gráfico de Bland e Altman para para as áreas $\leq 10 \mathrm{~cm}^{2}$ mensuradas pela pesquisadora (area1log) e pelas avaliadoras (area9log), transformadas logaritmicamente; b) Gráfico de Bland e Altman para as áreas $>10 \mathrm{~cm}^{2}$ mensuradas pelo (area1log) e pelas avaliadoras(area9log), transformadas logaritmicamente, Santa Maria, Rio Grande do Sul, Brasil, 2015. 


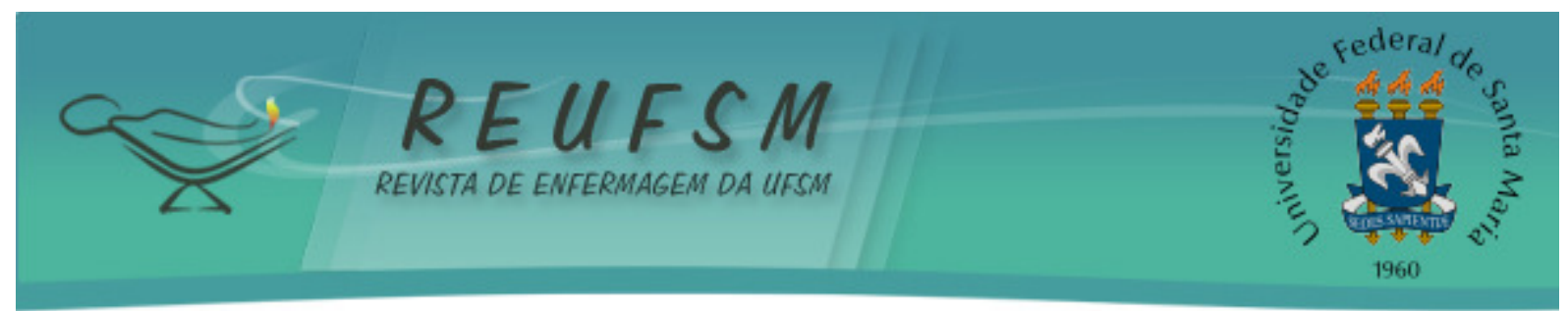

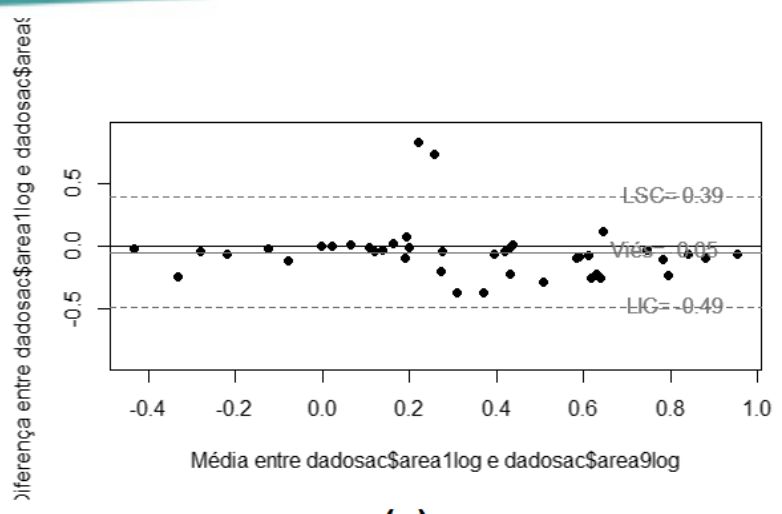

(a)

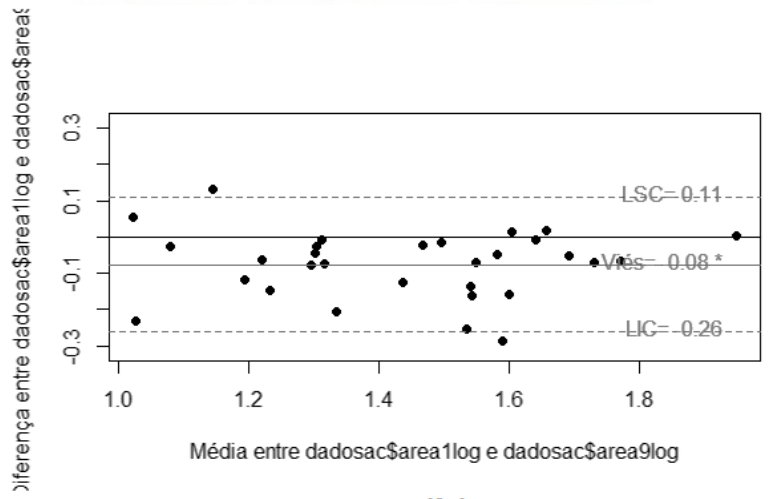

(b)

\section{DISCUSSÃO}

Os tamanhos das úlceras apresentaram grande amplitude (as áreas mensuradas variaram de 0,3 a $89,1 \mathrm{~cm}^{2}$ ) e as mensurações não apresentaram diferença estatisticamente significativa. Outros estudos, que caracterizaram os pacientes com úlcera venosa em atendimento ambulatorial, também encontraram grande amplitude entre os tamanhos das feridas. ${ }^{18-20}$

Destaca-se que houve forte correlação e reprodutibilidade entre as medidas. No entanto, o software parece ser mais preciso quando utilizado para mensurar feridas grandes (com área $>10$ $\mathrm{cm}^{2}$ ), pois os limites de concordância são clinicamente aceitáveis. Entretanto, ao analisar as feridas pequenas (com área $\leq 10 \mathrm{~cm}^{2}$ ), os limites de concordância são clinicamente inaceitáveis.

$\mathrm{Na}$ literatura, foram encontrados estudos sobre a avaliação de métodos de mensuração de feridas. Um estudo ${ }^{21}$ comparou o Image tool com o softwareAutoCAD®, não encontrando diferença estatisticamente significativa entre as mensurações realizadas com os dois softwares.

Também, três técnicas de mensuração para úlceras por pressão foram comparadas (régua, acetato, planimetria digital), as quais apresentaram forte concordância para medir a área de feridas pequenas, com formato circular e margens regulares. Quando as feridas apresentam formato irregular, os autores recomendam a utilização do acetato ou planimetria digital em detrimento da régua, ${ }^{6}$ corroborando o uso de fotografias e softwares para a mensuração de feridas.

Frente à atual realidade e preocupação com o uso de recursos financeiros cada vez mais escassos na saúde, as questões econômicas carecem ser consideradas ao escolher uma tecnologia ou tratamento mais apropriado. ${ }^{18,22-23}$ Nesse contexto, o Image tool, por ser um software gratuito, não apresentando custo de compra ou licença para seu uso, sendo uma alternativa importante na mensuração de área de úlceras venosas. 


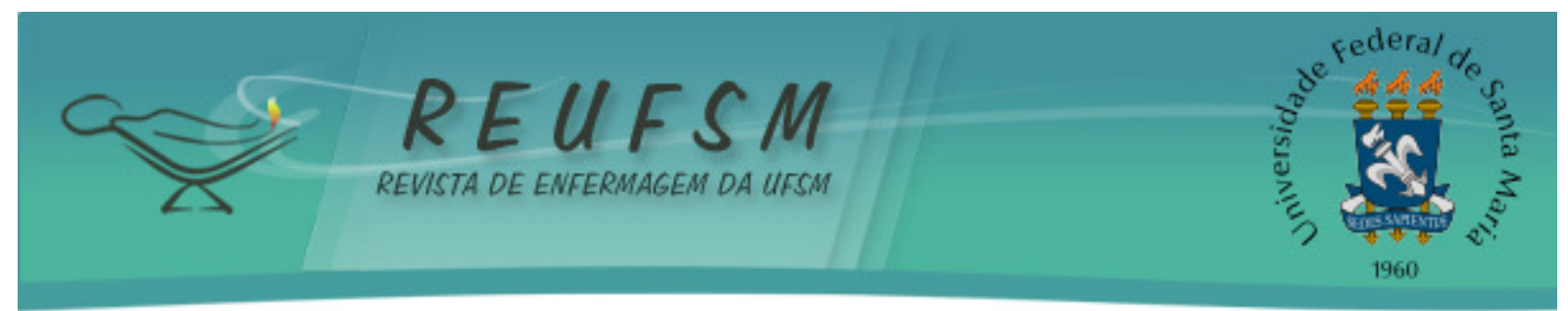

Uma das limitações deste estudo diz respeito à delimitação das bordas da ferida, a qual foi realizada de forma manual e dependia do conhecimento individual das avaliadoras acerca da definição de leito, superfície e bordas. Um estudo comparou as técnicas manual e automática de demarcação das bordas para cálculo de área pelo software ImageJ para avaliação de lesões por pressão, demonstrando que, apesar de haver correlação, houve pouca concordância. Nesse sentido, sugerem-se estudos que comparem estas duas técnicas de delimitação. ${ }^{24}$

\section{CONCLUSÃO}

Concluiu-se que houve correlação intraclasse $(\rho=0,97)$ e correlação de concordância ( $\rho=$ 0,95) excelentes entre as medidas das úlceras venosas mensuradas pela pesquisadora e pelas avaliadoras por meio do uso do software Image tool. Portanto, foi observada reprodutibilidade do uso do software para mensuração de área de úlceras venosas, sendo mais preciso para mensurar úlceras venosas com área $>10 \mathrm{~cm}^{2}$, recomendando-se o uso deste para a mensuração de feridas superficiais.

O estudo apresentou algumas limitações: o tipo de amostragem (não probabilística); a ausência de treinamento sobre conceitos básicos referentes à avaliação de feridas, como a definição do leito e bordas; o uso de avaliação bidimensional.

Desta forma, sugerem-se novos estudos com o referido software, analisando diferentes tipos de feridas crônicas e delimitação de bordas manual ou automática.

\section{REFERÊNCIAS}

1. O’Donnel Jr TF, Passman MA, Marston WA, Ennis WJ, Dalsing M, Kistner RL, et al. Management of venous leg ulcers: Clinical practice guidelines of the Society for Vascular Surgery® and the American Venous Forum. J Vasc Surg. 2014;60(2S):3S-59S.

2. Dias T, Costa I, Melo M, Torres S, Maia E, Torres G. Quality of life assessment of patients with and without venous ulcer. Rev Latinoam Enferm. 2014;22(4):576-81.

3. Medeiros ABA, et al. Associação dos fatores socioeconômicos e clínicos e o resultado integridade tissular em pacientes com úlceras. Rev Gaúcha Enferm. 2016 [acesso em 2017 maio 31];37(1):e54105. Disponível em: http://www.scielo.br/pdf/rgenf/v37n1/0102-6933rgenf-37-1-1983-144720160154105.pdf.

4. Dantas DV, Dantas RAN, Costa IKF, Torres GV. Protocolo de assistência a pessoas com úlceras venosas: validação de conteúdo. Rev RENE. 2013;14(3):588-99.

5. Dantas DV, Dantas RAN, Araújo RO, Vasconcelos QLDAQ, Costa IKF, Torres GV. Proposta de protocolo para assistência as pessoas com úlceras venosas. Rev Enferm UFSM. 2013;3(Esp.):618-26. 


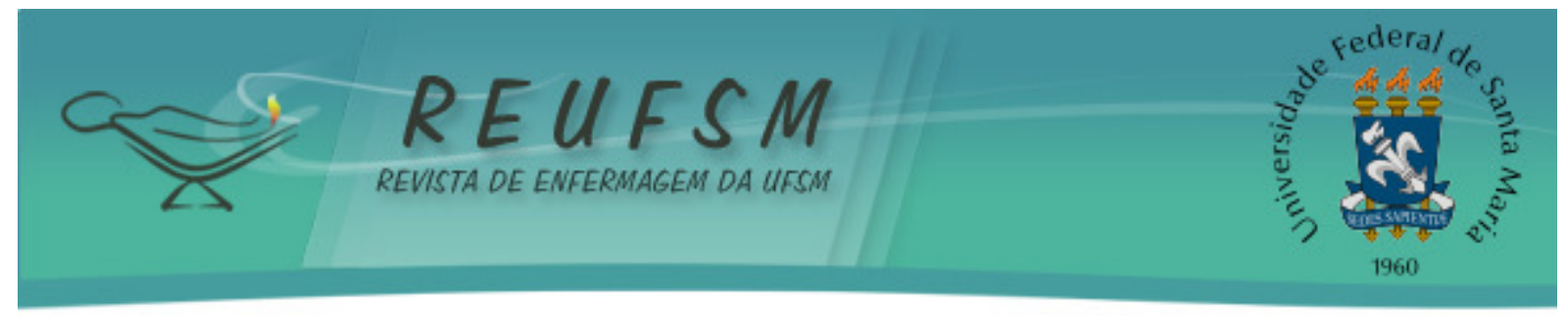

6. Dantas DV, Torres GV, Dantas RA N. Assistência aos portadores de feridas: caracterização dos protocolos existentes no Brasil. Ciênc Cuid Saúde. 2011;10(2):366-72.

7. Gonçalves M, Rabeh S, Terçariol C. The contribution of distance learning to the knowledge of nursing lecturers regarding assessment of chronic wounds. Rev Latinoam Enferm. 2015;23(1):e122.

8. Sousa A, Vasconcelos J, Soares M. Software Image Tool 3.0 as an instrument for measuring wounds. Rev Enferm UFPE. 2012;6(10):e2569.

9. Eberhardt T, Kessler M, Soares R, Lima S, Fonseca G, Rodrigues L. Mensuração de feridas: revisão da literatura. Ciência \& Saúde (Porto Alegre). 2015;8(2):79-84.

10. Bilgin M, Günes U. A comparison of 3 wound measurement techniques: effects of pressure ulcer size and shape. J Wound Ostomy Cont Nurs. 2013;40(6):590-3.

11. Lazarus G, Kirsner R, Zenilman J, Valle M, Margolis D, Cullum N, et al. Clinical interventions for venous leg ulcers: Proposals to improve the quality of clinical leg ulcer research. Wound Rrepair Regen. 2016;24(5):767-74.

12. Pereira MG. Epidemiologia: teoria e prática. Brasília: Guanabara Koogan; 1995.

13. Hulley S, Cummings S, Browner W, Grady D, Newman T. Delineando a pesquisa clínica: uma abordagem epidemiológica. $4^{\mathrm{a}}$ ed. Porto Alegre: Artmed; 2015.

14. Mandeville P. El coeficiente de correlaciòn de concordancia de Lin. Cienc UANL. 2007;(1):91-4.

15. Lin L. A concordance correlation coefficient to evaluate reproducibility. Biometrics. 1989;45(1):255-68.

16. Bland J, Altman D. Measuring agreement in method comparison studies. Stat Methods Med Res. 1999;8(2):135-60.

17. Malaquias S, Bachion M, Sant'Ana S, Dallarmi C, Lino Junior R, Ferreira P, et al. People with vascular ulcers in outpatient nursing care: a study of sociodemographic and clinical variables. Rev Esc Enferm USP. 2012;46(2):302-10.

18. Sant'ana S, Bachion M, Santos Q, Nunes C, Malaquias S, Oliveira B. Úlceras venosas: caracterização clínica e tratamento em usuários atendidos em rede ambulatorial. Rev bras enferm. 2012;65(4):637-44.

19. Borges E, Amorim I, Carvalho D. Características dos pacientes com ulcera venosa atendidos nas Unidades de Atenção Primária de Nova Lima, Minas Gerais. Rev Estima. $2014 ; 12(1)$.

20. Eberhardt T, Lima S, Lopes L, Borges E, Weilller T, Fonseca G. Measurement of the area of venous ulcers using two software programs. Rev Latinoam Enferm. 2016;24:e2862.

21. Shetty R, Sreekar H, Lamba S, Gupta AK. A novel and accurate technique of photographic wound measurement. Indian J Plast Surg. 2012;45(2):425-9.

22. Bilgin M, Günes UY. A comparison of 3 wound measurement techniques. J Wound Ostomy Continence Nurs. 2014;40(6):590-3. 


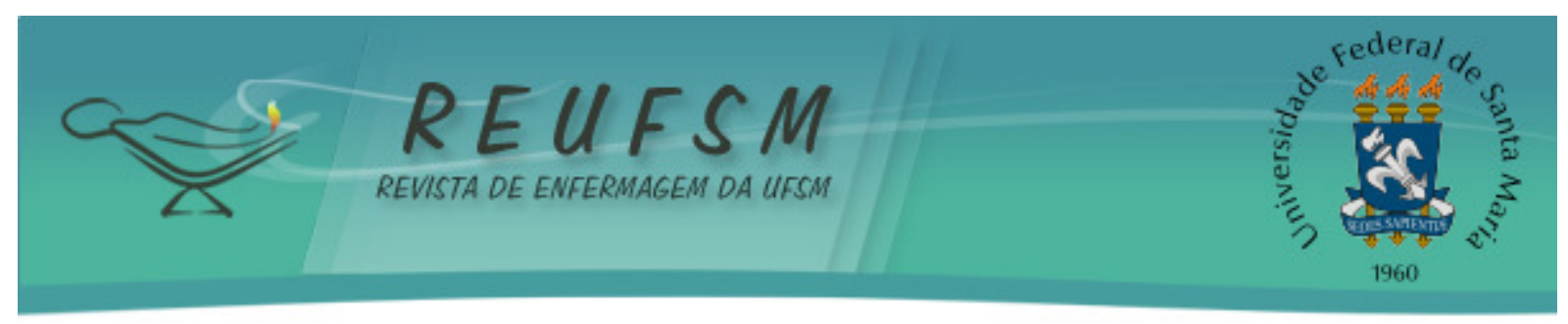

23. Salmona KBC, Santana LA, Neves RS, Guadagnin RV. Estudo comparativo entre as técnicas manual e automática de demarcação de borda para avaliação de área de úlceras por pressão. Enferm Foco. 2016;7(2):42-6.

Data de submissão: 10/06/2017

Data de aceite: $29 / 08 / 2017$

Autor correspondente: Thaís Dresch Eberhardt

Endereço: Av. Roraima n ${ }^{\circ}$ 1000, Cidade Universitária, Bairro Camobi, Santa Maria, RS, Brasil, CEP: 97105-900.

E-mail: thaiseberhardt@gmail.com

\section{AGRADECIMENTOS}

Bolsa demanda social Coordenação de Aperfeiçoamento de Pessoal de Nível Superior (CAPES). Bolsa Programa de Iniciação Científica (PROIC) - Hospital Universitário de Santa Maria (HUSM). 